\title{
SIMULATING EQUIPMENT-BASED CONSTRUCTION OPERATIONS USING VIRTUAL ENVIRONMENTS
}

\author{
KHALID K. NAJI \\ Assistant Professor, Department of Civil Engineering \\ College of Engineering, University of Qatar, Doha-Qatar, \\ P.O. Box: 2713, Email: knaji@qu.edu.qa
}

\begin{abstract}
Construction simulation using virtual environments is a hot topic that gained attention during the last ten years. Although there is an ongoing research investigating the effectiveness and usefulness of virtual environments in construction simulation, this technique never reached the general construction industry. This is mainly due to two reasons: the lack of knowledge and the high cost involved. This paper briefly presents a historical background of the techniques used in construction simulation based on visual interactive graphical techniques, and introduces a new methodology for simulating equipment-based construction operations using virtual environments on personal computer. Additionally, a case study of a virtual environment for a constructed facility is demonstrated.
\end{abstract}

Keywords: virtual reality, virtual environment, construction simulation, computer aided design.

\subsection{BACKGROUND}

Different simulation techniques assisted many construction engineers in making vital decisions related to construction operations. Nevertheless, in 1976 Hurrion was the first person to address the need of combining computer graphics and user interaction into the simulation process. Based on Hurrion's work the Visual Interactive Simulation (VIS) technique began to gain more focus in construction simulation [1]. Later Hurrion developed SIMON, a simulation language to support VIS. His first package was called VISION, which was further modified to allow more flexibility in user-simulation interaction [2]. By the late 1970s simulation packages were extended to a variety of graphical modes and customizable icons to represent the machines and entities that interact in the system. With advances in computer technology, companies improved the input and modeling capabilities of VIS giving the birth to VIM (Visual Interactive Modeling) [3]. However, it was not until the 1990s when the VIS technique included the use of real-time computer graphics and virtual environments in construction simulation.

In the literature there is no clear information about when and where Virtual Reality (VR) began to be injected in construction simulation; however, some of the early work was done in late 1970s. The Department of Construction Management and Engineering at the University of Reading, in the United Kingdom, developed a 3-D, computer-aided building design tool, called CABD, which allowed the creation and manipulation of solid models representing building components [4]. Following that work, the Cybernetics Virtual Reality Research Group at Reading University developed a fully immersive virtual reality interface which can be used to walk through building designs developed on CABD [4].

In 1994, in the United States, a program called the Interactive Visualizer Plus Plus (IV++) was developed to model building components and simulate construction operation at the Georgia Institute of Technology by Opdenbosch [5]. In his Ph.D. thesis Opdenbosch presented a new way to model and simulate construction operations in virtual environments [6]. The key element in the IV++ methodology was to utilize real-time computer graphics in the simulation using virtual environments. Additionally, the IV++ provided a new way of user-simulation interaction by allowing the user to participate in the 3-D computer-generated environment. Although the IV++ was developed on a UNIX-based computing platform, Opdenbosch forecasted that his technique would be possible to be adapted on the personal computer in few years. 
This paper presents a new methodology for simulating equipment-based construction operations using an object-oriented approach, and based on realtime computer graphics. Although the methodology presented here utilizes state-of-the-art techniques in developing virtual environments, it has been demonstrated on the Windows NT operating environment.

\subsection{VIRTUAL ENVIRONMENTS IN CONSTRUCTION}

There is a number of terms describing what might be considered as a virtual environment; however, a virtual environment describing a construction operation could be defined as a 3-D computergenerated environment representing the construction site, building components, surrounding environment material objects, labor objects, and machine objects as shown in figure 1. Additionally, the user must have the ability to interact with the environment, and control both the viewing conditions and the objects participating in the environment [6]. What governs this user-environment interaction and control is a set of predefined behaviors stored in the environment and applied to all objects within the environment [7].

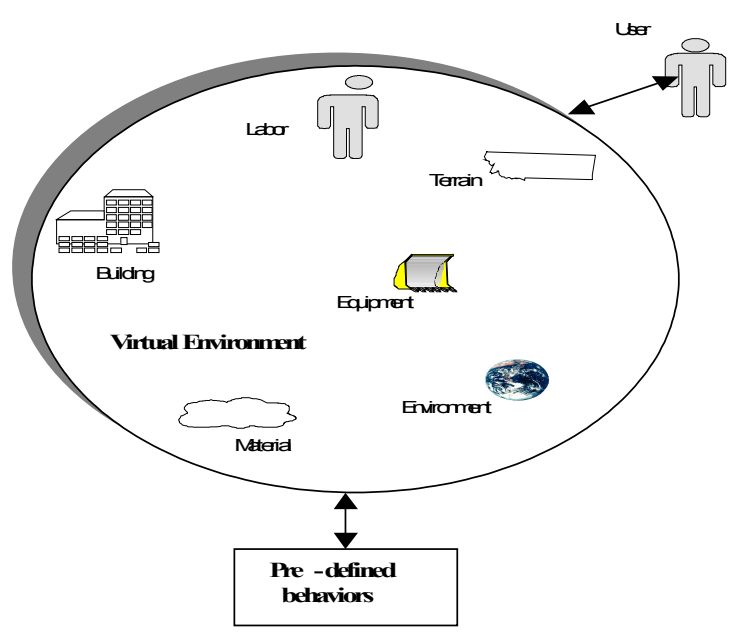

Figure 1: The Structure of a Virtual Environment

\subsection{THE SIMCON ENVIRONMENT}

This section introduces a virtual environment, named SIMCON, developed to graphically simulate equipment-based construction operations. The development of SIMCON was based on an object-oriented approach and real-time computer graphics [7]. The environment was built using WorldUP ${ }^{1}$, a modeling and authoring package which facilitates the creation of distributable virtual environments [8]. The structure of modules developed to control the environment is presented in figure 2. The graphical user interface (GUI) of SIMCON forms the basic building block by which the user communicates with two modules environment, and machine control through a main control module.

The machine control module allows the user to load, unload or control four different machine prototypes: truck, tower crane, mobile crane and an excavator. These machine prototypes are defined in SIMCON as objects where more than one machine could be loaded at any time and participate in the environment.

The environment module facilitates the user control of the environment such as: loading a building component, activating a sensory device, initializing a viewing port and so on. Since any meaningful simulation of construction operations should include the construction site [9]; therefore, the environment module allows the user to load either a flat or any other imported irregular terrain objects into the environment.

The there main modules developed in SIMCON were developed using the scripting language, Basic Script ${ }^{2}$, inside the WorldUP development environment. Similarly, all other GUI objects such as: windows, message boxes, loading/saving dialog boxes etc., were developed using the scripting language.

Features of the SIMCON's environment are as follows:

- The ability to import any graphical objects such as: 3D site layouts and site objects from CAD programs using a number of drawing exchanging formats (e.g. DXF, 3DS...etc).

- The ability to import any building geometry from CAD programs using the drawing exchange format (DXF) in order to simulate the geometric components of a building under construction.

- The ability to graphically simulate laborbased operations.

- The ability to graphically simulate equipment operations using 4 equipment prototypes (truck, tower crane, mobile crane and an excavator).

\footnotetext{
${ }^{1}$ WorldUP is a registered trademark of Sense8 Corp. and Engineering \& Imagination Inc.

${ }^{2}$ Basic Script is a registered trade mark of Summit Technologies.
} 
- Full user-machine control using input devices (2D mouse, 3D mouse, serial joystick, and the keyboard).

- Immersive 3D environment using head mount display (HMD) and data gloves.

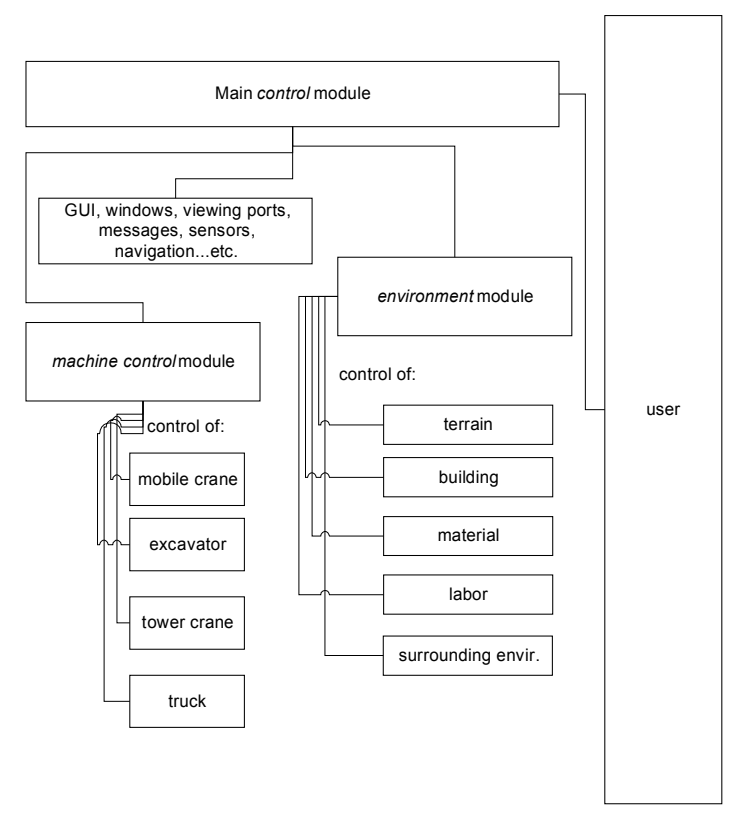

Figure 2: Modules Structure of SIMCON

\subsection{OBJECTS' BEHAVIORS IN SIMCON}

As mentioned earlier, the only way to control any visual object within the virtual environment is to define a set of pre-defined behaviors. Behaviors like how a certain object should respond upon selection, activation or when a certain event is fired were pre-defined in the main control module of SIMCON. Some of these behaviors are described in more details in the next sub sections.

\subsection{Equipment Dynamics}

In SIMCON each machine has the ability to move on either a flat or an irregular terrain. That was done using a general terrain-following algorithm, which force the machine to be on the terrain and follow the topography every simulation cycle. Based on the terrain topology, the final variable that describes the movement of the machine along its local z-axis is its velocity. In SIMCON machines are controlled using a non-autonomous mode pre-defined in the machine control module. Thus by using a keyboard or the serial joystick, each machine can be translated by a unit translation vector along its local z-axis.

\subsection{Collusion Detection}

In SIMCON the system keeps a list of names of every object that is active within the simulation. This list is checked every simulation cycle against collusion. For example, if a tower crane object is trying to move an object from one point on the terrain to another point inside a building object, a collusion event is fired and the system displays a message warning the user that two objects are going to collide with each other. Another example, if a truck object is moving between two different points on the terrain and the truck collided with another machine, a labor object or another machine object, the collusion event is fired and the system warns the user both in a text-based and a voice-based annotations.

\subsection{Labor Dynamics}

Simulating the dynamic movement of workers between two different locations is one of the most complex tasks that traditional simulation techniques do not provide visually [10]. In SIMCON labor movement between two different locations is simulated using a General Problem Solving (GPS) algorithm. This reactive algorithm consists of 5 different actions as follows:

- find the target and move towards it

- if an obstacle exists on the path, move opposite from the target direction

- rotate towards an obstacle-free orientation

- move forward

- find the target and move towards it

The same algorithm is applicable for $\mathrm{n}$ number of stations since the visual object representing a worker resource will move between two points only at a time.

\subsection{CASE STUDY}

The case study presented in this paper is the original case on which SIMCON was developed. It is a $\$ 33$ million dollar project within the University of Florida's main campus initiated in June 1996 and was scheduled to finish in July 1998 [7]. Figure 3 presents the CAD model of the Brian Institute Building (UF project \#BR-147).

Based on a lump sum bid, the total cost of the project included design, construction and the installed medical equipment for a total area equals 
30,000 sq ft. Two multistory buildings were proposed to surround the north and the west sides of the allocated area for the project, where two small roads existed on the east and the south sides. According to the main contractor, McDevitt Bovis, construction space was a critical problem in the project specially during the first $65 \%$ of the project's schedule.

According to the project manager several equipment-based operations were delayed since day one of the project. According to the company's scheduling data, these operations were delayed because of their nature of being on the critical path of the project, which is quite natural. This is due to the fact that at the beginning of any construction project, many of the scheduled operations involves the utilization of heavy equipment as a primary resource. Once the building skeleton is built, laborbased operations become more involved and intensive.

As a result of this investigation, the virtual environment SIMCON was developed to test the ability to detect any interference of machines with the building model, the surrounding environment or between different machines in the site.
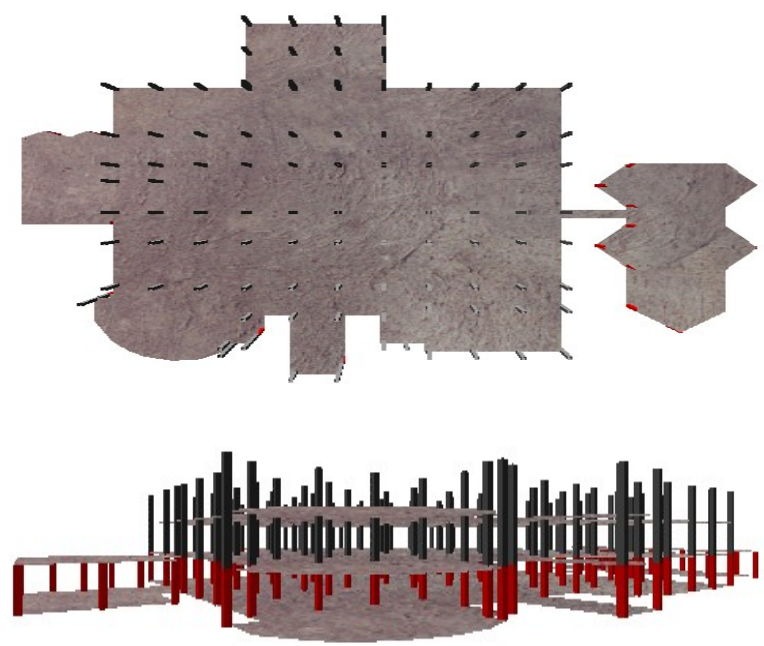

Figure 3: The Brain Institute Building CAD Model

By default SIMCON assumes a regular flat terrain of the construction site. However, if the actual terrain topography of the construction site has some irregularities the user can use the environment module to load the terrain CAD file into SIMCON. In this case study, a flat terrain object was assigned to the environment. Then, the same module was used to load the building CAD model, which was developed using Autocad $^{3}$ R. 14, and imported to the invironment.

Additionally, environment objects describing the surroundings of the case study such as: roads, cars, trees, material patches, fence etc., were loaded into the environment. Figure 4 illustrates the virtual environment (SIMCON) after all visual objects have been loaded into the environment.

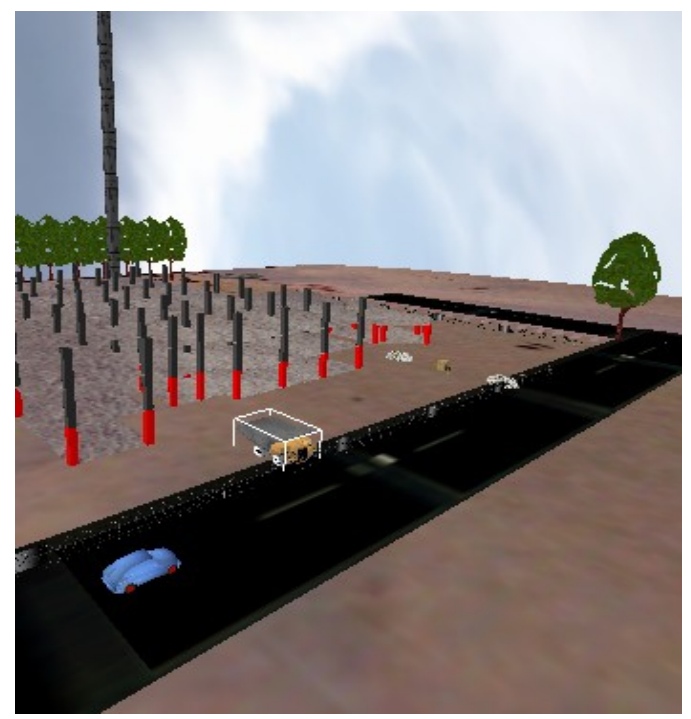

Figure 4: The Brain Institute Virtual Environment

In this case study four different machine prototypes were used in the simulation. These prototypes are: truck, mobile crane, tower crane and an excavator. One of the most advanced features of SIMCON is the ability to load $\mathrm{n}$ number of machines during the simulation and change the user control between them. Figure 5 presents an early stage of the simulation where the boom of the tower crane object was used in a non-autonomous mode to place a patch of material over the building object.

\footnotetext{
${ }^{3}$ Autocad is a registered trade mark of Autodesk Corp.
} 


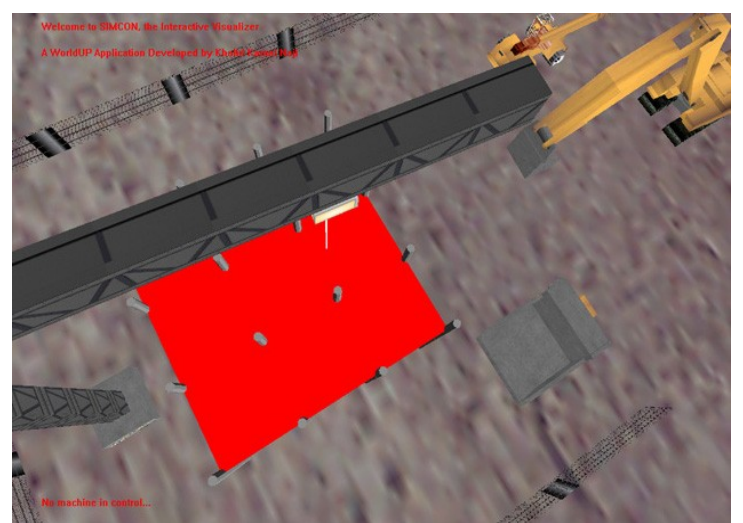

Figure 5: The Tower Crane Object in SIMCON

Similarly, figure 6 shows the boom of the mobile crane object where it is approaching the same location of the material object, in a non-autonomous mode, to move it to a different location on the terrain.

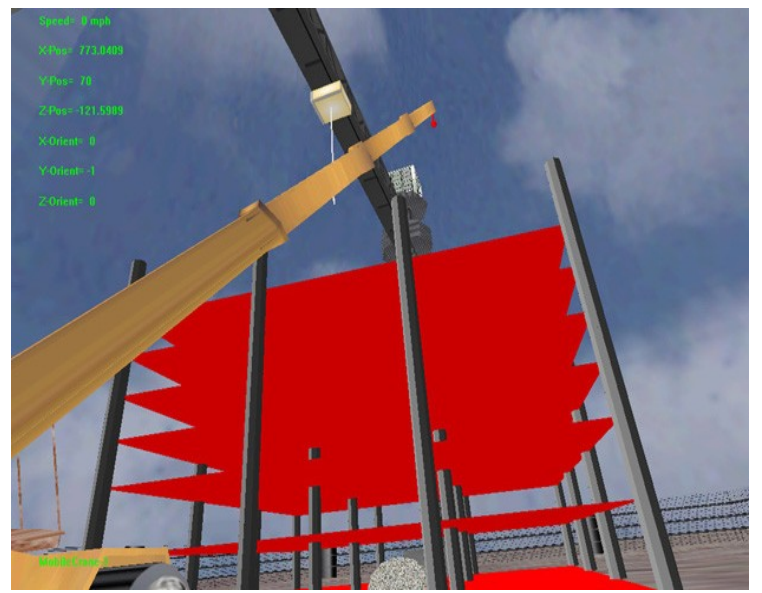

Figure 6: The Mobile Crane Object in SIMCON

As mentioned earlier, one of the most powerful features of SIMCON is the ability to simulate labor operations. For this case study the environment module was used to initialize a crew of two workers moving between two different material stations as shown in figure 7. In this case an obstacle object was located at the middle distance of the two material objects. During the simulation process the object representing the labor crew was able to detect this obstacle object and corrected its path whenever it was traveling from one station to the other. This operation simulated a true material handling operation taped from the real construction site.

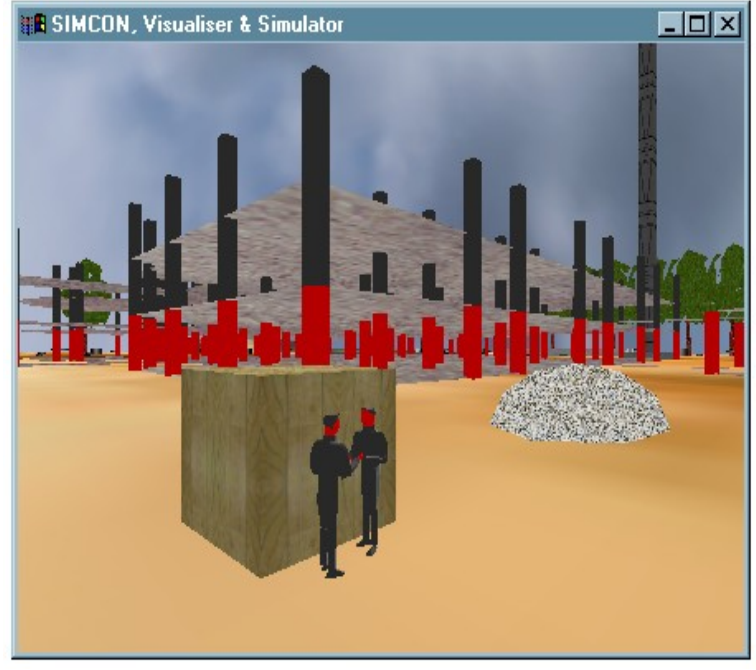

Figure 7: Simulation of Labor-Based Operation

Once all of the objects representing the surrounding environment of the case study such as: labor crews and machines, a truck-based material handling operation was activated in SIMCON. This operation was activated to investigate three features in SIMCON:

- The ability to detect any interference of a machine object with a labor object.

- The ability to record and report the time associated with an operation.

As shown in the schematic sketch (figure 8) the truck-based operation was activated between stations $\mathrm{S} 1$ and S2. Once the truck object is moved from S1 towards S2 and collides with either points N1 or N1 representing material objects SIMCON displays a collusion-detection message to the user. If a similar scenario occurred between the truck object and the labor object or the object representing the obstacle, the same warning message appears to the user. Additionally, a voice-based warning message is triggered and activated. To add more realism to the simulation SIMCON sets the velocity of the truck to zero and orient it towards a new path.

Similarly, when the truck is moving from points S1 to S2 and hits any of the site boundary objects, (e.g. fence objects), SIMCON detects this type of collusion and displays a text and a voicebased warning messages that a site-based object and a machine are colliding with each other. As shown in figure 8 a site gate object was added to the model representing the case study. This model does not appear in the environment as a physical graphical object; however, it consists of two small entities (nodes) located at the starting and the ending points of the site border objects (fence). 
Another type of collusion detection mechanism in SIMCON could be demonstrated if the truck object collides with one of the objects representing the traffic flow around the site (e.g. cars). This collusion detection mechanism was predefined in the system as machine-environment collusion type.

The last feature of SIMCON that was demonstrated in this example is the ability to measure the required operation time. Assuming that the user initialized the timer function when the truck was ready to depart from point S1 to S2, once the truck approached point S2 SIMCON displays a textbased message indicating the complete operation time.

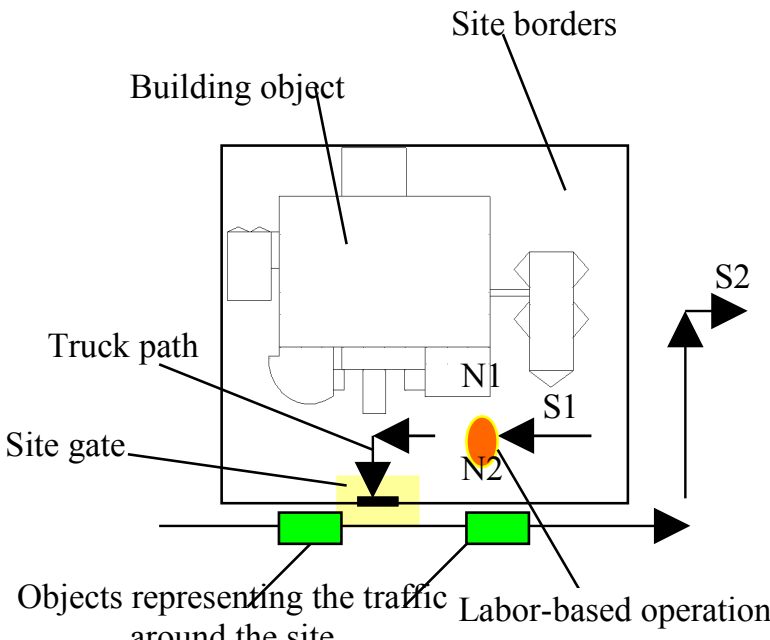
around the site

Figure 8: A schematic Sketch of the Truck Operation

As mentioned earlier SIMCON was developed such that it allow the user to be fully immersed in the simulation. The user can set different viewing ports as well as initiating different sensory devices such as: 3D mouse, joystick, data glove or a head mount display (HMD). In this case study full immersive situation was tested which yielded to a high level of user-simulation interaction. This immersive situation was reached by using a hmd, a 2D mouse to freely navigate through the environment and a data glove for selecting/deselecting simulation objects. Additionally, different viewing port positions were tested such as the 360 degree front-machine camera presented in figure 9 .

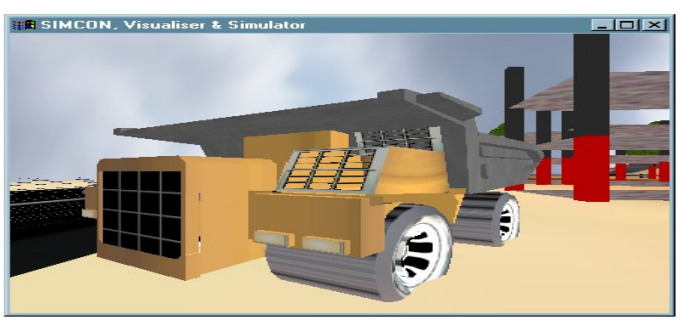

Figure 9: The Environment viewed from FrontMachine Camera

\subsection{CONCLUSION}

The work presented in this paper demonstrated a new methodology for simulating equipment-based construction operations on an inexpensive computer platform, the Windows environment. Additionally, it highlighted the basic elements required to build a virtual construction environments based on an object-oriented graphical technique. The workability of the methodology was demonstrated by a case study from the real world. It is worthwhile to be noted here that even though SIMCON was developed based on a real case study, it can be adopted to similar case studies in the future.

\section{REFERENCES}

[1] O'Keefe R. M., "What is Visual Interactive Simulation? (And is there a methodology for doing it right?)", Winter Simulation Conference, NY, pp. 461-464, 1987.

[2] Bell P. C., and O'Keefe R. M., "Visual Interactive Simulation (VIS) history, recent developments, and major issues", Simulation 49, no. 3, pp. 109-116, 1987.

[3] Bishop J. L., and Balci O., "General purpose visual simulation system: a functional description", Winter Simulation Conference, pp. 504-512, 1990.

[4] Warwick K., Gray J., and Roberts D., Virtual reality in engineering, The Institution of Electrical Engineers, London, UK, 1993.

[5] Opdenbosch A., "Design/Construction Processes Simulation in Real Time Object-Oriented Environments", Ph.D. Thesis, Georgia Institute of Technology, 1994.

[6] Opdenbosch A., and Baker N., "Simulation of construction operations in virtual interactive 
environments", ASCE proceedings, pp. 1435-1442, NY, 1995.

[7] Naji K. K., "The Development of a Virtual Environment for Simulating Equipment-Based Construction Operations in Real-Time ObjectOriented Systems", Ph.D. Dissertation, The University of Florida, 1997.

[8] WorldUp User's Guide, May Release, Sense8 Corporation, Mill Valley, CA, 1996.

[9] Oloufa A. A., "Modeling and Simulation of Construction Operations", Automation in Construction, pp. 351-359, 1993.

[10] Rodriquez W., Opdenbosch A., Ferrier A., and Patterson W., "Interactive Visualizer (IV): Injecting reality in the design process", ASEE/EDGD 1993 Mid-Year Conference, San Francisco, Jan. 9-12, pp. 1-8, 1993. 